\title{
QCD Correlations in Multiple Gluon Bremsstrahlung
}

\section{W. A. Horowitz ${ }^{a, *}$}

${ }^{a}$ Department of Physics, University of Cape Town,

Private Bag X3, Rondebosch 7701, South Africa

E-mail: wa.horowitz@uct.ac.za

We compute for the spectrum for emitting 1 and 2 soft and collinear gluons from a hard scattering process in full QCD. This result is important because 1) all current energy loss calculations assume QED-like independent emissions of multiple gluons, which obviously misses all correlations from the non-Abelian nature of QCD, and 2) the average high- $p_{T}$ parton emits $\sim 3$ gluons as it escapes the medium. QCD correlations are therefore critical for any realistic comparison of theoretical predictions to experimental data on jets and jet substructures in heavy ion collisions. These calculations additionally provide a benchmark for jet Monte Carlo algorithms.

HardProbes 2020

1-6 June 2020

Austin, Texas

\footnotetext{
${ }^{*}$ Speaker
} 


\section{Introduction}

With heavy ion collisions, we wish to explore experimentally and theoretically the non-trivial, many-body dynamics of QCD. High momentum probes provide the most direct probes of the fundamental degrees of freedom in the quark-gluon plasma created in the heavy ion collisions at the Relativistic Heavy Ion Collider (RHIC) and the Large Hadron Collider (LHC). In the weakly-coupled picture of the energy loss of these high- $p_{T}$ probes, the scattering of the high- $p_{T}$ probe off of inmedium quasiparticles leads to the stimulated emission of soft and collinear gluon bremsstrahlung [1]. Reasonable calculations estimate that the number of gluons emitted in a typical propagation of a high- $p_{T}$ particle through the QGP medium is of the order of 3 [2]. Energy loss calculations implemented in phenomenological energy loss models have so far included the effects of multiple gluon emission through a Poisson-like convolution that assumes independence between the emissions of gluons [3]; i.e. the calculations assume that the non-Abelian corrections to a QED-like treatment of multiple emissions is small. We would like to test this assumption with a calculation of the correlations in multiple gluon emission in heavy ion collisions. As a first step towards that derivation, we will compute the multiple gluon emission process, including QCD correlations, for the multiple gluon emissions associated with a hard scattering process in vacuum, which we present here. Further, we'll use what are known as spinor helicity techniques, which are a new way of computing scattering amplitudes that are often far more efficient than the usual techniques employed in quantum field theoretic calculations [4-8].

\section{A Model Problem}

For the case of interest in this work, one finds that the full amplitude can be decomposed as $[4,5]$

$$
\begin{aligned}
& \mathcal{A}_{\bar{q} g^{n} q g^{m}}^{\text {tree }}\left(p_{\bar{q}}^{h_{\bar{q}}}, k_{1}^{h_{1}}, \ldots, k_{n}^{h_{n}}, p_{q}^{h_{q}}, \ell_{1}^{h_{1}}, \ldots, \ell_{m}^{h_{m}}\right)= \\
& g_{s}^{n+m+2} \sum_{\sigma \in S_{n}, \tau \in S_{m}}\left(T^{a_{\sigma(1)}} \cdots T^{a_{\sigma(n)}} T^{a_{\tau(1)}} \cdots T^{a_{\tau(m)}}\right)_{\alpha \beta} \times \\
& A_{\bar{q} g^{n} q g^{m}}^{\text {tree }}\left(p_{\bar{q}}^{h_{\bar{q}}}, \sigma\left(k_{1}^{h_{1}}\right), \ldots, \sigma\left(k_{n}^{h_{n}}\right), p_{q}^{h_{q}}, \tau\left(\ell_{1}^{h_{1}}\right), \ldots, \tau\left(\ell_{m}^{h_{m}}\right)\right) .
\end{aligned}
$$

where the sum is performed over all possible permutations of the $n+m$ gluons and $g_{s}$ is the usual strong coupling constant, $\alpha_{s} \equiv g_{s}^{2} / 4 \pi$. In this expression, $A_{\bar{q} g^{n} q g^{m}}^{\text {tree }}$ is known as a partial or color-stripped amplitude, which is gauge invariant. In the literature, various other names have been given to this gauge invariant component, such as color-ordered amplitude and dual amplitude [4,5]. Each partial amplitude in the sum corresponds to a particular color flow, which can be naively thought as the ordering in which the gluons are emitted. That the above decomposition is valid is a highly non-trivial result and depends on trading structure constants for color generators through the relationship $\tilde{f}^{a b c} \equiv i \sqrt{2} f^{a b c}=\operatorname{tr}\left(T^{a}\left[T^{b}, T^{c}\right]\right)$ [4]. The color kinematic decomposition is especially powerful because the computation of the partial amplitude is significantly easier than the full amplitude. In particular, partial amplitudes have an extremely compact and simple form when they maximally violate helicity conservation. These MHV amplitudes have exactly two negative helicity external particles while all other external particles have positive helicity. This generalizes 
to the notion of $\mathrm{N}^{k} \mathrm{MHV}$ amplitudes when $(k+2)$ external particles have negative helicity. The case where exactly two particles have positive helicity while all the other particles have negative helicity are called anti-MHV, $(\overline{\mathrm{MHV}})$.

Using the spinor helicity formalism and the Britto-Cachazo-Feng-Witten (BCFW) on-shell recursion relation [11], one can show that a general MHV partial amplitude for a process which involves an arbitrary number of gluons and a quark-antiquark pair is given by [5]

$$
A_{\bar{q} q g^{n}}^{M H V}\left(p_{\bar{q}}^{-}, p_{q}^{+}, 1^{+}, \ldots, k^{-}, \ldots, n^{+}\right)=\frac{\left\langle p_{\bar{q}} k\right\rangle^{3}\left\langle p_{q} k\right\rangle}{\left\langle p_{\bar{q}} p_{q}\right\rangle\left\langle p_{q} 1\right\rangle \cdots\left\langle n p_{\bar{q}}\right\rangle} .
$$

The partial amplitude for our MHV helicity configuration expressed in Eq. (2) is remarkably simple and only a function of the angle brackets. The $\overline{\mathrm{MHV}}$ partial amplitude can be derived from Eq. (2) by swapping all helicities, which results in changing the angle brackets into square brackets and multiplying by an overall sign depending on the number of external legs. Thus $\left|A\left(\left\{h_{i}\right\}\right)\right|^{2}=\left|A\left(\left\{-h_{i}\right\}\right)\right|^{2}$.

Armed with Eq. (2) we may immediately compute the dominant soft and collinear emission of a single bremsstrahlung gluon radiation associated with $q g \rightarrow q g$ scattering. The relevant amplitude is

$\mathcal{A}\left(1^{-} 5^{+} 2^{+} 3^{-} 4^{+}\right) \simeq g^{3}\langle 13\rangle^{3}\langle 23\rangle\left(T^{5} T^{3} T^{4} \frac{1}{\langle 15\rangle\langle 52\rangle\langle 23\rangle\langle 34\rangle\langle 41\rangle}+T^{5} T^{4} T^{3} \frac{1}{\langle 15\rangle\langle 52\rangle\langle 24\rangle\langle 43\rangle\langle 31\rangle}\right)$,

where we have used the shorthand $T^{i} \equiv T^{a_{i}}$. We are able to drop the additional contributions to the amplitude $\mathcal{A}\left(1^{-} 5^{+} 2^{+} 3^{-} 4^{+}\right)$from Eq. (1) for the following reason. We are interested in radiation soft and collinear to the outgoing quark, which is particle 1 . Since angle brackets can be thought of as something akin to the square root of a dot product, $\langle 15\rangle$ is therefore very small when particle 5 , the associated bremsstrahlung radiation for the hard scattering $q g \rightarrow q g$ process, is soft and collinear to the outgoing quark, particle 1 . None of the other permutations of the three gluons will have a $\langle 15\rangle$ in the denominator and will therefore be small in comparison to the two terms included in Eq. (3).

Comparing to the $q g \rightarrow q g$ amplitude, one readily sees for radiation emitted soft and collinear to the outgoing quark that

$$
\mathcal{A}\left(1^{-} 5^{+} 2^{+} 3^{-} 4^{+}\right) \simeq g T^{5} \frac{\langle 12\rangle}{\langle 15\rangle\langle 52\rangle} \mathcal{A}\left(1^{-} 2^{+} 3^{-} 4^{+}\right) .
$$

The same logic follows for all other helicity configurations of the hard scattering subprocess. We therefore have that

$$
\left\langle\left|\mathcal{A}_{5}\right|^{2}\right\rangle=2 g^{2} \tilde{C}_{F} \frac{s_{12}}{s_{15} s_{52}}\left\langle\left|\mathcal{A}_{4}\right|^{2}\right\rangle \equiv\left|\mathcal{J}_{g}^{(1)}\right|^{2}\left\langle\left|\mathcal{A}_{4}\right|^{2}\right\rangle
$$

where $\left\langle\left|\mathcal{A}_{4}\right|^{2}\right\rangle$ is given by the square of the $q g \rightarrow q g$ amplitude and we have defined the single gluon emission kernel $\left|\mathcal{J}_{g}^{(1)}\right|^{2}$ in the second line. Notice in the first line the critical overall factor of 2 due to the $\overline{\mathrm{MHV}}$ contributions. 
One may write the outgoing parton momenta in the usual way for high energy QCD processes and find that the emission spectrum derived above gives

$$
\frac{d N_{g}^{(1)}}{d^{3} k} \simeq C_{F} \frac{\alpha_{s}}{\pi^{2}} \frac{1}{x} \frac{1}{k_{\perp}^{2}}
$$

in exact agreement with the single gluon radiation spectrum of [12] associated with the scattering of a hard quark, in the limit of a massless quark.

Now consider the same process with two gluons soft/collinear with the outgoing fermion. We have that to leading order in kinematics, i.e. for terms enhanced by inverses of $\langle 15\rangle,\langle 16\rangle$, and/or $\langle 56\rangle$,

$$
\begin{aligned}
& i \mathcal{M}\left(1_{\bar{q}}^{-} 2_{q}^{+} 3^{-} 4^{+} 5^{+} 6^{+}\right) \\
& \quad=i g^{4}\langle 14\rangle^{3}\langle 24\rangle\left[\frac{T^{3} T^{4} T^{5} T^{6}}{\langle 12\rangle\langle 23\rangle\langle 34\rangle\langle 45\rangle\langle 56\rangle\langle 61\rangle}+\frac{T^{3} T^{4} T^{6} T^{5}}{\langle 12\rangle\langle 23\rangle\langle 34\rangle\langle 46\rangle\langle 65\rangle\langle 51\rangle}+(3 \leftrightarrow 4)\right]
\end{aligned}
$$

Simplification leads to

$$
i \mathcal{M}\left(1_{\bar{q}}^{-} 2_{q}^{+} 3^{-} 4^{+} 5^{+} 6^{+}\right)=i \mathcal{M}_{4}\left(1_{\bar{q}}^{-} 2_{q}^{+} 3^{-} 4^{+}\right) \mathcal{S}_{2}(5,6),
$$

where the two particle soft factor is

$$
\mathcal{S}_{2}(5,6) \equiv \sum_{\sigma} T^{\sigma(5)} T^{\sigma(6)} S_{2}(\sigma(5), \sigma(6)), \quad S_{2}(5,6) \equiv \frac{g^{2}\langle 12\rangle}{\langle 25\rangle\langle 56\rangle\langle 61\rangle} .
$$

One may straightforwardly square the amplitude to find

$$
\left|\mathcal{S}_{2}(5,6)\right|^{2}=g^{4}\left[\tilde{C}_{F}^{2} \frac{s_{12}^{2}}{s_{15} s_{25} s_{16} s_{26}}+\tilde{C}_{F} \tilde{C}_{A} \frac{s_{12}}{s_{56}} \frac{\operatorname{tr}(\not \$ \not 1 \phi)}{s_{15} s_{25} s_{16} s_{26}}\right] .
$$

The NMHV contribution is non-trivial to calculate. One finds the result

$$
\begin{array}{r}
\left|\mathcal{S}_{2, N}\right|^{2}=g^{4}\left[\tilde{C}_{F}^{2} \frac{s_{12}^{2}}{s_{15} s_{16} s_{25} s_{26}}+\frac{\tilde{C}_{F} \tilde{C}_{A}}{s_{56}^{2} s_{15} s_{16} s_{25} s_{26} s_{156}^{2} s_{256}^{2}}\left\{2 s_{15} s_{16} s_{25} s_{26}\left(s_{15} s_{26}-s_{16} s_{25}\right)^{2}\right.\right. \\
\left.\left.+s_{12} s_{56} s_{156} s_{256}\left[\operatorname{tr}(1 \not \not \not \varnothing 6)\left(s_{16} s_{26}+s_{15} s_{25}\right)-4 s_{15} s_{16} s_{25} s_{26}\right]\right\}\right] .
\end{array}
$$

Combining the MHV and NMHV results yields

$$
\begin{aligned}
\left|\mathcal{S}_{2, t o t}\right|^{2}=\frac{2 \tilde{C}_{F} s_{12}}{s_{15} s_{52}} & \frac{2 \tilde{C}_{F} s_{12}}{s_{16} s_{62}}+\frac{2 \tilde{C}_{F} \tilde{C}_{A}}{s_{56}^{2} s_{15} s_{16} s_{25} s_{26} s_{156}^{2} s_{256}^{2}}\left\{2 s_{15} s_{16} s_{25} s_{26}\left(s_{15} s_{26}-s_{16} s_{25}\right)^{2}\right. \\
+ & \left.s_{12} s_{56} s_{156} s_{256}\left[\operatorname{tr}(1 \not \not \not \not \varnothing)\left(s_{16} s_{26}+s_{15} s_{25}+s_{156} s_{256}\right)-4 s_{15} s_{16} s_{25} s_{26}\right]\right\} .
\end{aligned}
$$




\section{Conclusions}

We presented here the one and two gluon soft and collinear bremsstrahlung associated with hard quark-gluon scattering in vacuum as computed using spinor helicity techniques. The one gluon emission derivation is trivial and reproduces exactly the long-known results from QCD. The two gluon emission result, Eq. (12), requires significant additional effort to compute the next-to-maximal helicity violating contributions. It's unclear how exactly to adapt spinor helicity techniques to the hot and dense quark-gluon plasma environment or whether there is enough gain in efficiency to allow for computing multiple gluon emission in this environment. There might be a useful application of the two gluon emission result in computing particle correlations in cooler environments such as $p+p$ and $p+A$ collisions.

\section{Acknowledgments}

The author gratefully acknowledges financial support from the South African National Research Foundation, the South Africa-CERN Collaboration, and from the University of Cape Town.

\section{References}

[1] A. Majumder and M. Van Leeuwen, Prog. Part. Nucl. Phys. 66, 41 (2011) [arXiv:1002.2206 [hep-ph]].

[2] M. Gyulassy, P. Levai and I. Vitev, Phys. Lett. B 538, 282 (2002) [nucl-th/0112071].

[3] N. Armesto et al., Phys. Rev. C 86, 064904 (2012) [arXiv:1106.1106 [hep-ph]].

[4] L. J. Dixon, “A brief introduction to modern amplitude methods," arXiv:1310.5353 [hep-ph].

[5] J. M. Henn and J. C. Plefka, Lect. Notes Phys. 883, pp.1 (2014).

[6] H. Elvang and Y. t. Huang, "Scattering Amplitudes in Gauge Theory and Gravity."

[7] A. N. Rasoanaivo and W. A. Horowitz, arXiv:1712.06292 [hep-ph].

[8] T. R. Rabemananajara and W. A. Horowitz, J. Phys. Conf. Ser. 889, no. 1, 012021 (2017). doi:10.1088/1742-6596/889/1/012021

[9] S. Catani and M. Grazzini, Nucl. Phys. B 570, 287 (2000) [hep-ph/9908523].

[10] M. E. Peskin and D. V. Schroeder, "An Introduction to quantum field theory."

[11] R. Britto, F. Cachazo, B. Feng and E. Witten, Phys. Rev. Lett. 94, 181602 (2005) [hepth/0501052].

[12] M. Djordjevic and M. Gyulassy, Nucl. Phys. A 733, 265 (2004) [nucl-th/0310076]. 